\title{
Potential Applications of the Escherichia coli Heat Shock Response in Synthetic Biology
}

\author{
Joana L. Rodrigues ${ }^{1, \star}$ and Lígia R. Rodrigues ${ }^{1}$
}

The Escherichia coli heat shock response (HSR) is a complex mechanism triggered by heat shock and by a variety of other growth-impairing stresses. We explore here the potential use of the $E$. coli HSR mechanism in synthetic biology approaches. Several components of the regulatory mechanism (such as heat shock promoters, proteins, and RNA thermosensors) can be extremely valuable in the creation of a toolbox of well-characterized biological parts to construct biosensors or microbial cell factories with applications in the environment, industry, or healthcare. In the future, these systems can be used for instance to detect a pollutant in water, to regulate and optimize the production of a compound with industrial relevance, or to administer a therapeutic agent in vivo.

The Potential of the E. coli HSR

The HSR is an essential mechanism to protect cells against adverse conditions including heat shock (HS), viral infection [1], UV irradiation [2], high alcohol concentrations [1], heavy metals [3], oxidative stress [4], and recombinant protein production [5]. These stress factors increase the levels of denatured proteins which can be harmful to the cell owing to changes in their biological activities. Therefore, when cells are exposed to stress conditions they respond by synthesizing socalled heat shock proteins (HSPs) such as chaperones and proteases. Chaperones assist in protein folding if the proteins are misfolded or unfolded [6]. Proteases eliminate the proteins denatured by high temperatures that the chaperones were not able to refold [7]. This ensures that the problems created by stress conditions are reduced and toxic aggregates are not formed.

The HSR mechanism is highly complex (Box 1) and has great potential to be used in synthetic biology (see Glossary) approaches. The development of a library of countless and designable biological parts with predictable behavior that can be easily integrated into complex genetic systems is one of the key drivers of synthetic biology [8-11]. For example, owing to increased pathway complexity, it is important to expand the range of available inducible promoters and other regulatory parts. Several players in the E. coli HSR mechanism can be very useful to create a toolbox of well-characterized biological parts [e.g., HS promoters, genes, proteins, and RNA thermosensors (RNATs)] that can be used to construct devices and systems, such as biosensors and microbial cell factories (Figure 1), with applications in healthcare, industry, or the environment. In this paper we focus on the potential of the E. coli HSR in conjunction with synthetic biology strategies in several biotechnological applications.

\section{Development of Synthetic Biology Parts, Devices, and Systems Based on the E. coli HSR}

The E. coli HSR can be useful in the design of new biological parts, devices, and systems for a variety of applications such as those detailed below. The HS promoters constitute an example of a biological part of increasing interest because these can trigger the expression of a desired gene in response to a temperature increase or other stressful conditions [12,13]. The HSR
Trends

Synthetic biology is an exciting field that aims to redesign biological systems for different applications.

The development of a toolbox containing well-characterized biological parts is essential to create systems that function in predictable ways to develop innovative solutions for various challenges.

Bacteria have been synthetically designed to carry out specific tasks. The components of $E$. coli HSR mechanism, such as heat shock promoters, proteins, and RNA thermometers, may be extremely valuable in the creation of organisms with novel functionalities.

Biosensors/reporter bacteria are being developed to detect and measure the presence of harmful chemicals in food, soil, and water, or to detect a specific disease. Biological parts may also enable advances in the genetic control of metabolic pathways with relevant industrial applications.

${ }^{1}$ Center of Biological Engineering University of Minho, Campus de Gualtar, 4710-057 Braga, Portugal

*Correspondence: joana.joanalucia@deb.uminho.pt (J.L. Rodrigues). 
Box 1. The E. coli HSR Mechanism

In E. coli the HSR is controlled positively at the transcriptional level $[91,92]$ by a protein that binds to RNA polymerase (RNAP) allowing it to bind to DNA and recognize HS promoters. This protein is the product of the rpoH gene, the transcription factor $\sigma^{32}$ [1]. This transcription factor is necessary to induce HS gene expression. In steady-state conditions $\left(30-37^{\circ} \mathrm{C}\right)$, the concentration of $\sigma^{32}$ is very low and limits HS genes transcription. When the temperature increases to $42^{\circ} \mathrm{C}$ the HSR is induced via an increase in $\sigma^{32}$ protein levels and possibly its activity $[91,92]$. This type of $\sigma^{32}$ regulation is fast, and allows $E$. coli cells to mount a prompt and efficient response to the abrupt HS. However, this mechanism only has a small effect on $\sigma^{32}$ synthesis because, in stress conditions at $42^{\circ} \mathrm{C}, r p o H$ transcription does not increase significantly [91,92]. At higher temperatures or under different stress conditions, the effect in $\sigma^{32}$ synthesis can be more pronounced because the regulatory region of the $\mathrm{rpoH}$ gene is complex and contains several promoters that are dependent on different transcription factors $[4,91,92]$. The second mechanism of $\sigma^{32}$ synthesis regulation concerns the translation of $r p o H$ mRNA [4,91,92]. At low temperature several mRNA regions anneal, leading to the formation of an mRNA secondary structure (stem-loop) that inhibits protein synthesis and acts as a RNAT [22]. This structure is responsible for repressing $r p o H$ translation at steady-state because the Shine-Dalgarno (SD) sequence is only partially exposed and the start codon is inaccessible. This prevents the formation of the ribosomal initiation complex [22,27]. Translation induction on exposure to heat takes place as a result of partial melting of the secondary structure caused by the temperature increase, allowing ribosome binding and translation initiation. In addition to $\sigma^{32}$ transcriptional and translational control, $\sigma^{32}$ activity and degradation are also regulated by HSPs (negative feedback control) [93-96]. The chaperones involved in $\sigma^{32}$ regulation are mainly the DnaK and GroE systems (DnaK/DnaJ/GrpE and GroEL/GroES) $[1,91,92,97]$, and one of the proteases responsible for $\sigma^{32}$ degradation is FtsH. Because FtsH lacks robust unfolding activity $[98,99]$, the DnaK system has an essential role in enhancing $\sigma^{32}$ recognition and stabilization for $\mathrm{FtsH}$ by binding to the factor, preventing it from binding to RNAP, thus indirectly increasing $\sigma^{32}$ factor degradation and allowing homeostatic balance $[93,94,98-100]$.

mechanism has been widely studied and the genes involved have been identified by microarrays using different HSR triggering methods. Table 1 summarizes the most highly induced HS genes [1,14-18]. In addition, $\sigma^{32}$-dependent promoters have been identified using $\mathbf{C h I P}$ Chip [15]. This technique determines the strength of in vivo binding of $\sigma^{32}$ to the corresponding promoters instead of the induction ratio of gene expression. Therefore, different results may be obtained depending on the stimulus applied. For example, when the temperature is very high $\left(T \geq 50^{\circ} \mathrm{C}\right)[18]$, the HSR is higher, faster, and is controlled at the transcriptional level not only by $\sigma^{32}$ but also by $\sigma^{24}$. In addition, several studies have shown that the HS genes are also controlled by other transcription factors (e.g., $\sigma^{54}$ ) [19]. For example, the ibpAB operon contains a $\sigma^{32}$-dependent promoter upstream of the ibpA gene, and a $\sigma^{54}$-dependent promoter upstream of the $i b p B$ gene. Thus, ibpB can be translated from two sets of transcripts. To study the HS genes that only depend on $\sigma^{32}$, this factor was overexpressed from a plasmid $[1,14]$. In these studies the induction ratios were in some cases lower than when HS was used as the HSR trigger [16-18]. This occurred because the HSR was initiated as a result of increased levels of $\sigma^{32}$, and was not due to misfolded proteins produced as a consequence of elevated temperature. Because chaperones are not needed to help in protein folding, they act as negative modulators and kidnap $\sigma^{32}$ that afterwards is degraded by proteases (Box 1). Therefore, the induction of the HS genes is not as high. In addition, induction may be distinct in terms of kinetics (fast/slow) or magnitude (strong/weak) [14], and this can be relevant when choosing a promoter for synthetic biology applications. Some HS promoters allow a high induction ratio and high expression levels during $\mathrm{HS}$, a high induction ratio but relatively low expression during HS, or a medium induction ratio and high expression levels [17].

Depending on the intended purpose, different types of promoters may be chosen. In cases where the goal is to produce a specific compound for industrial purposes, it is important to take into account not only the desired product concentration but also its toxicity to the cell. A low concentration may be insufficient for the intended purpose, but when too high it can damage the cells [17]. Therefore, extremely high expression is not always desirable because it can lead to an excess of misfolded proteins. In synthetic biology and metabolic engineering 'more is not

\section{Glossary}

Biosensor: biological device that detects and quantifies biologically relevant molecules and outputs a detectable signal in response. ChIP-chip: chromatin immunoprecipitation (ChIP) coupled with microarray analysis (Chip) to characterize the interactions of protein with genomic DNA. Induction ratio: the difference between the expression level before and after the HSR is triggered. Metabolic burden: influence of engineered pathways on the distribution of the available resources (energy molecules or carbon building blocks) in the host cell.

Metabolic flux: the rate of metabolite flow through a metabolic pathway that is regulated by the enzymes in the pathway.

Microarray: hybridization-based technology that enables the relative concentrations of nucleic acid sequences in a sample to be measured.

Synthetic biology: the design and construction of new biological entities such as enzymes, genetic circuits, and cells, or the redesign of existing biological systems (www.ebrc.org/ what-is-synbio). 


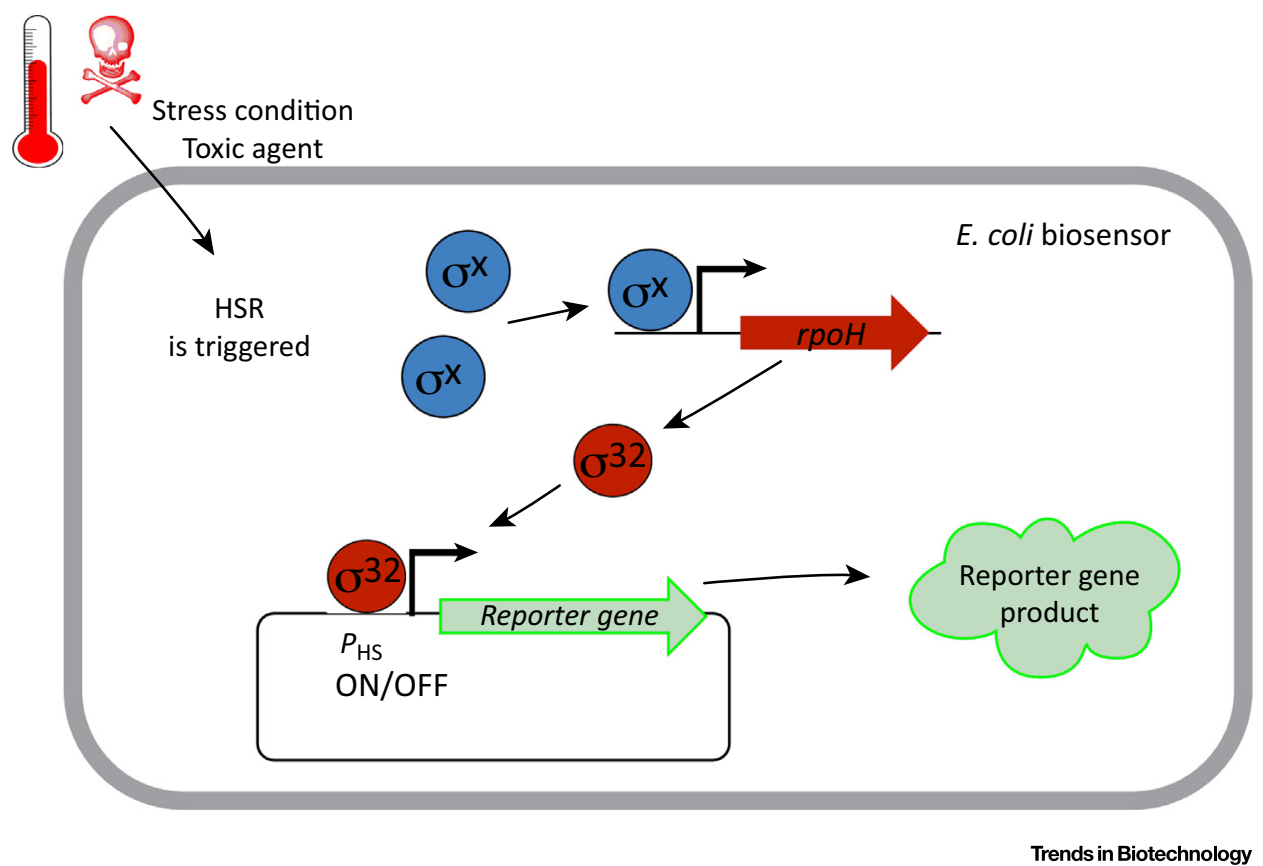

Figure 1. Schematic Representation of an E. coli Biosensor. The sensing module is composed by a heat shock (HS) promoter sequence (e.g., dnaK, ibpA, grpE) that is activated after the E. coli heat shock response (HSR) is triggered by different stress conditions such as HS or high concentrations of chemicals (e.g., polluted environment). The activation of the HS promoters triggers the expression of a reporter gene (e.g., lux, luc, gfp, lacZ). The response can be measured by fluorimetry, colorimetric assay, confocal microscopy, or flow cytometry depending on the reporter used. These biosensors can be used in different applications with high sensitivity. In addition, this type of design can also be used to express a protein of interest to produce for example a therapeutic compound in industry (e.g., antibiotic).

always better', and in pathways with multiple enzymes it is important to fine-tune expression levels and metabolic flux to limit the accumulation of intermediary byproducts and to maximize end-product yield $[13,20]$.

In addition to the level of expression, it is important to take into account the induction ratio. When expression levels are high and induction ratios are relatively low, expression levels can already be high before the HSR is triggered. Depending on the application, this might constitute a problem. For example, for in vivo applications such as treatments that should be administered in a specific area after an external stimulus (see the 'Therapeutic Applications' section), promoters that allow a low induction ratio might not be appropriate because they are already normally active at normal body temperature $\left(37^{\circ} \mathrm{C}\right)$. In these cases, promoters with lower leakage at low temperature may be preferable even if the expression levels after triggering HSR are not as high. Because different applications have different needs, it is crucial to construct libraries of engineered tandem promoters with different strengths and induction ratios for testing in each specific situation. It is important to test the promoters in the exact conditions intended because, as shown in Table 1, the expression/induction ratio may greatly vary because of the complexity of the HSR mechanism. In addition, ribosome binding sites should be tested and optimized to obtain a translational efficiency that matches the expression levels previously observed by microarray analysis $[13,17]$.

The HSR can also be very useful in the design of RNATs (Figure 2) [21], which can find numerous applications in synthetic biology where protein expression level is the central goal 
Table 1. The $\sigma^{32}$-Regulon Members, Including Significantly Expressed Escherichia coli HS Genes

\begin{tabular}{|c|c|c|c|c|c|c|c|}
\hline \multirow[t]{2}{*}{ Blattner number } & \multirow[t]{2}{*}{ HS gene } & \multicolumn{5}{|c|}{ Induction ratio of HS genes using different HSR triggers } & \multirow{2}{*}{$\begin{array}{l}\text { Binding strength of } \\
\sigma^{32} \text { to } \mathrm{HS} \text { promoter } \\
\mathrm{HS} 30^{\circ} \mathrm{C} \\
\text { to } 50^{\circ} \mathrm{C} \text {, } \\
10 \mathrm{~min}[15]\end{array}$} \\
\hline & & $\begin{array}{l}\mathrm{HS} 37^{\circ} \mathrm{C} \\
\text { to } 50^{\circ} \mathrm{C} \text {, } \\
5 \mathrm{~min}[18]\end{array}$ & $\begin{array}{l}\text { Overexpression } \\
\text { of } \sigma^{32} \text { at } 37^{\circ} \mathrm{C} \text {, } \\
5 \min [1]\end{array}$ & $\begin{array}{l}\text { Overexpression } \\
\text { of } \sigma^{32} \text { at } 30^{\circ} \mathrm{C} \text {, } \\
10 \min [14]\end{array}$ & $\begin{array}{l}\mathrm{HS} 30^{\circ} \mathrm{C} \text { to } \\
42^{\circ} \mathrm{C} \\
5 \text { min }[16]\end{array}$ & $\begin{array}{l}\mathrm{HS} 37^{\circ} \mathrm{C} \\
\text { to } 45^{\circ} \mathrm{C} \text {, } \\
5 \mathrm{~min}[17]\end{array}$ & \\
\hline b3686 & $i b p B$ & 327.2 & 20.5 & 13.4 & 128 & 224.76 & a \\
\hline b3687 & $i b p A$ & 297.4 & 12.9 & 13.4 & 59.7 & 55.68 & 20.4 \\
\hline b0015 & dnaJ & 85.3 & 2.3 & 18.2 & 8 & 7.08 & a \\
\hline b4142 & groEs & 77.5 & 4.8 & 9.1 & 13 & 6.79 & 27.4 \\
\hline b0014 & dnak & 58.5 & 4.8 & 18.2 & 8 & 7.59 & 111.8 \\
\hline b4140 & $f X S A$ & 50.7 & 7.4 & 4.4 & $-^{\mathrm{b}}$ & 16.89 & 46.8 \\
\hline b4143 & groEL & 37.9 & 7.6 & 9.1 & 7.5 & 7.9 & a \\
\hline b2592 & $c / p B$ & 36.5 & 16.6 & 21.5 & 11.3 & 9.17 & 31.1 \\
\hline b1829 & $h t p X$ & 36.1 & 5.2 & 12.1 & - & 9.82 & 49 \\
\hline b0966 & yccV & 34.3 & 5.7 & 2.4 & - & 3.54 & 15.7 \\
\hline b0473 & $h t p G$ & 33.8 & 31.8 & 18.4 & 14.9 & 13.02 & 29.1 \\
\hline b1060 & yсеP & 25.5 & 17.9 & 6.9 & - & 9.44 & 20.9 \\
\hline b2614 & grpE & 24.1 & 10.5 & 14.2 & 8.6 & 4.95 & 17.8 \\
\hline b0439 & Ion & 20.3 & 3.6 & 9.3 & 6.1 & 6.76 & 30.3 \\
\hline b1322 & $y c j F$ & 19.8 & 7.5 & 6.9 & 6.5 & 23.82 & a \\
\hline b3498 & prlc & 16.7 & 8.3 & 9.2 & - & 9.17 & 28.3 \\
\hline b3932 & $h s / V$ & 16.2 & 6.9 & 15.5 & 6.5 & 8.07 & 43.4 \\
\hline b3635 & mutM & 12.2 & 10.1 & 12.4 & - & 21.1 & $<3.0$ \\
\hline b3931 & $h s / U$ & 10.3 & 10.2 & 15.5 & 7 & 6.58 & a \\
\hline b0492 & $y b b N$ & 9.9 & 9.6 & 11.6 & 7 & 11.15 & 59.9 \\
\hline b0660 & ybez & 9.6 & 4.7 & 7.4 & 3.2 & 4.41 & 49.9 \\
\hline b3293 & $y h d N$ & 9.5 & $<2.0$ & 11.9 & 4.6 & 6.42 & 13.2 \\
\hline b3067 & $r p o D$ & 7.7 & 1.9 & 10.5 & - & 3.9 & 14.6 \\
\hline b0631 & $y b e D$ & 7.1 & 7.1 & 9.5 & - & 5.54 & 37.1 \\
\hline b1321 & $y c j x$ & 3.9 & 9.3 & 6.9 & 7.5 & 21.77 & 58 \\
\hline b1380 & $I d h A$ & 3.4 & 12.6 & 15.8 & - & 20.12 & 9 \\
\hline b0437 & $c / p P$ & 3.3 & $<2.0$ & 11.4 & - & 4.5 & 41.2 \\
\hline b0438 & $c / p X$ & 2.9 & 2.8 & 11.4 & - & 3.66 & a \\
\hline b1379 & hslJ & 2.7 & 9.7 & 15.8 & & 6.3 & a \\
\hline b1279 & ycis & $<5.0$ & 4.1 & 3.7 & - & 4.27 & 72.1 \\
\hline
\end{tabular}

${ }^{a}$ These genes are not directly downstream of $\sigma^{32}$ factor-dependent promoters because they are in an operon.

${ }^{\text {b}}$ The induction ratio is not mentioned because the study only refers to genes with an expression ratio higher than 2.5 -fold in both treatments tested (HS and canavanine).

$[22,21]$. RNATs are RNA sequences that contain the ribosome binding site and function by sequestering the Shine-Dalgarno (SD) sequence, and in some cases the start codon, in a stem-loop structure at low temperature, thereby inhibiting translation [19,23]. At high temperatures the secondary structure unfolds and liberates the SD sequence and the start codon, thus allowing translation of the downstream gene [24]. Most known RNATs in E. coli control the 
(A)

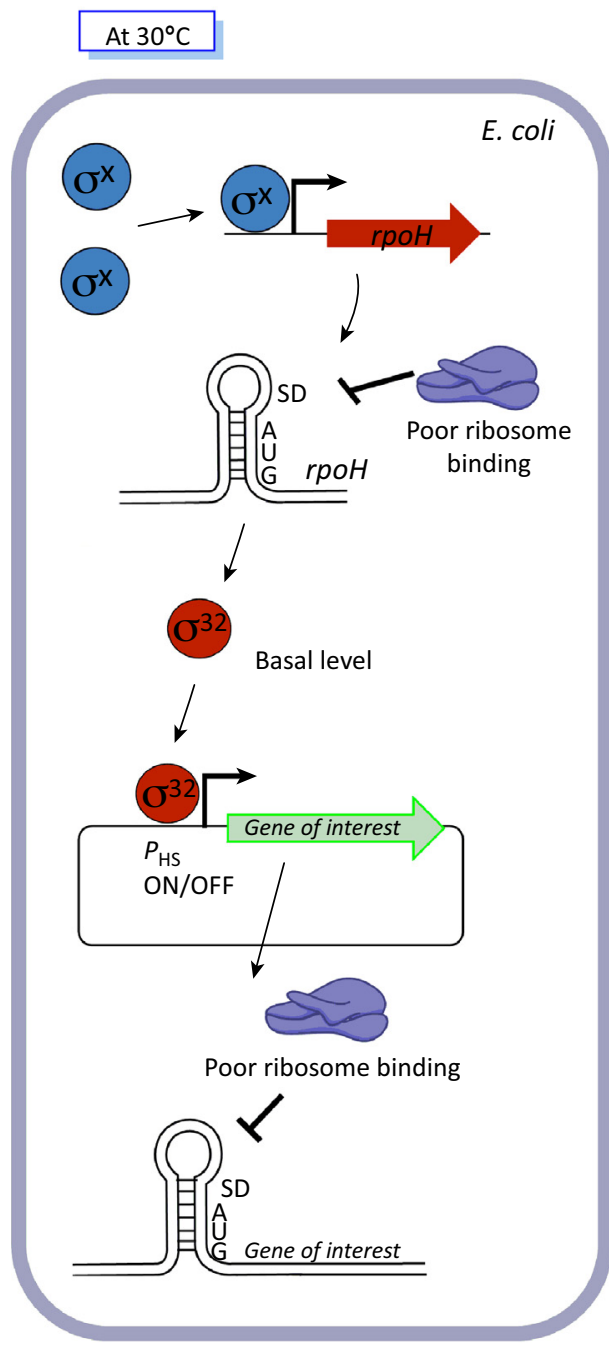

(B)

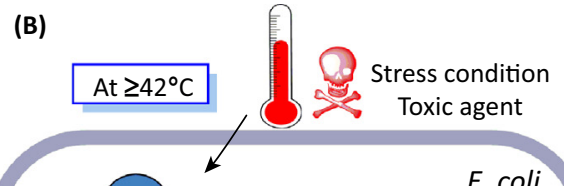

$\sigma^{x}$

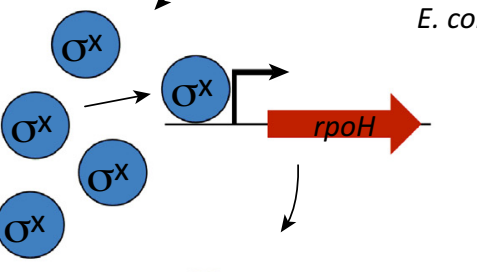

$\mathrm{rpoH}$

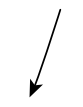

Increased level


Trends in Biotechnology

Figure 2. E. coli RNA Thermosensor (RNAT). (A) At low temperature, the RNAT traps the Shine-Dalgarno (SD) sequence and/or the translation initiation codon (AUG) by base pairing within a secondary structure. (B) When the temperature increases the secondary structure partially unfolds and translation initiation of the gene of interest takes place. In this specific example, a heat shock (HS) promoter is used. Secondary structures should be interpreted as merely illustrative.

synthesis of HSPs (e.g., rpoH and ibpAB transcripts) [22,19,23]. Although natural RNATs may be difficult to implement in engineered systems owing to low reusability or modularity and high complexity [21], they can be used as models to design smaller and simpler RNATs with a very tightly regulated 'OFF' state that can alleviate the promoter leakage problem previously mentioned. However, as with promoter design, the design of the optimal RNAT also depends on the final goal. RNATs with high protein expression in the ON state may be more suitable than RNATs that have low leakiness (low expression in OFF state) but also have low protein expression in the ON state [21]. Therefore, these biological parts can also be exploited as versatile control elements to respond to a predefined temperature, and can be implemented in complex genetic circuits to modulate expression in small- and large-scale settings [21,24,25]. 
Synthetic RNATs can be heat-inducible or heat-repressible, meaning that at high temperatures they induce or repress gene expression, respectively. Most natural occurring RNATs are heatinducible. However, heat-repressible RNATs have also been reported or designed [21,24]. Synthetic heat-repressible RNATs may be used in the future to downregulate pathways of product synthesis that redirect resources necessary for growth or survival during periods of stress that cause metabolic burden [21]. Tight shut-off is especially important when toxic products are synthesized [26]. This type of RNAT can also be explored as a reverse-genetic tool to study the function of essential genes [21] or to switch ON/OFF the expression of a toxic gene or a pathogen-killing toxin $[27,21]$. In addition, these genetic tools are the only known singlecomponent regulators of genetic expression $[24,28]$ that operate independently of transcription factors. Thus, they are easily transferable between different bacterial expression hosts and, consequently, are attractive modules in synthetic biology. Moreover, they do not require the addition of chemical inducers that are expensive and can be toxic $[8,22,26,29]$. Therefore, de novo design of these RNATs has recently received attention with the goal of creating a toolbox of temperature biosensors with different thresholds and sensitivities [21,26,28,30,31].

HSP genes, such as those encoding chaperones or other gene products that can increase the HSR when expressed (e.g., rpoH), can also be very useful parts in synthetic biology given their potential role in heterologous protein folding [5] (see the 'Industrial Applications' section).

\section{Potential Applications of the HSR Mechanism}

All the previously described genetic parts with predictable behavior (HS promoters, genes, and RNATs) can be useful not only in cell-based applications but also in cell-free systems [31]. In this section we discuss the potential of these genetic parts in synthetic biology strategies to develop solutions targeting important issues in different fields.

\section{Environmental Applications}

The use of HS promoters to initiate the desired response of a specific gene has been extensively studied. In E. coli, HS promoters have been used to construct biosensors or toxicity reporters. Whole-cell biosensors or reporter strains are easy to construct and are fast and reliable tools [32]. The expression of a reporter gene (e.g., encoding $\beta$-galactosidase, luciferase, or a fluorescent protein) is activated with a specific stimulus and can be useful to detect toxic compounds or to understand the behavior of bacteria when exposed to pollutants, xenobiotics, and other compounds that represent a threat to living organisms [32]. The HSR can be activated by different types of stress such as HS, nutrient limitation (amino acids), or the addition of IPTG (isopropyl $\beta$-D-1-thiogalactopyranoside), ethanol, phenol acetic acid, antifoam, or salt (osmotic shock), among others [17,32,33]. For example, the E. coli HS promoters of $d n a K$, ibpA, and grpE were fused to luciferase (luxCDABE operon) from Vibrio fischeri, which confers bioluminescent properties [34-36]. These biosensors could detect the presence of metals, solvents, and other pollutants (Figure 1). When these compounds were present in water, the HSR was activated and was detected by the presence of light. These biosensors were also used to assess compound toxicity and their ability to cause damage to proteins [35]. To monitor the presence of misfolded proteins and their aggregates during the expression of heterologous proteins, the lucA reporter gene encoding firefly luciferase was fused to the tandem promoter ibpfxs constructed using ibpAB and fxs A promoters [37]. The reporter unit with the tandem promoter gave higher expression levels than constructs based on the single promoters.

Green fluorescent protein (GFP) from Aequorea victoria has also been fused to HS promoters $(r p o H, c / p B, d n a K$, ibpA, and $f x S A)[17,32,33]$. Fusions of gfp and lux to the grpE HS promoter 
were compared, and lux was found to be more efficient as a reporter because the GFP fluorescence signal developed much more slowly and the induction ratio was lower [38]. However, fluorescent reporters are still widely used because the signal accumulates for hours whereas bacterial bioluminescence is relatively short-lived [38]. Aertsen and colleagues [39] fused GFP to dnaK, Ion, and clpPX promoters to study which genes are induced by high hydrostatic pressure, while Nemecek and colleagues [40] and Dragosits and colleagues [41] fused the dnaK and ibpA promoters to GFP or RFP (red fluorescent protein), respectively, to monitor the overburden of recombinant protein production in E. coli hosts.

Popovtzer and colleagues [42] designed an electrochemical nano-biochip to detect toxic substances in water. The gene encoding LacZ was fused to the dnaK and grpE promoters such that the production of $\beta$-galactosidase was induced in the presence of toxic chemicals (high concentrations of ethanol or phenol). By adding its substrate ( $p$-aminophenyl $\beta$-Dgalactopyranoside), $p$-aminophenol was produced and its oxidation created a detectable current. Sekhon and colleagues [43] also constructed a biosensor using LacZ gene as a reporter and $i b p A$ promoter, but in this case with a very effective color-readout sensing system. Exposure to high concentrations of ethanol induced $\beta$-galactosidase production that hydrolyzed the substrate ortho-nitrophenyl- $\beta$-galactoside (ONPG) into galactose and the yellow pigment ortho-nitrophenol (ONP). Eltzov and colleagues [44] used the luxCDABE operon fused to the grpE promoter to detect air pollution. The $E$. coli cells were immobilized in alginate in wells of a microtiter plate and exposed to toxic compounds (e.g., chloroform) in air. The biosensor was also adapted to a portable fiber optic biosensor, and the authors predicted that in the future it could be used as an air pollution alert system in an indoor environment. More recently, they optimized this approach by developing a biosensor comprising two parts: a non-disposable part (monitor) and a disposable part (bioluminescent bacteria immobilized in alginate pads) [45], and further adapted it for determining water toxicity [3]. These stress-specific E. coli biosensors enable multianalyte detection, high-throughput screening, portability, and real-time detection in a quick, easy, and affordable way $[42,43]$. Therefore, it is expected that this type of biosensors could be very useful not only in environmental point-of-care testing but also in healthcare and medical applications.

Industrial Applications

E. coli is one of the most widely used hosts for the production of heterologous proteins of commercial or therapeutic interest, including high-value products such as biofuels or drugs. Among many other advantages, E. coli can grow on inexpensive substrates and, because of extensive genetic knowledge and the large number of compatible molecular biology tools that are available, it can be easily genetically modified [10]. However, many eukaryotic proteins of interest such as therapeutic proteins (e.g., antibodies) have complex tertiary or quaternary structures that usually require post-translational modifications such as glycosylation, phosphorylation, and addition of fatty acid chains that are necessary to obtain correct folding and biological activity [29]. Therefore, the overexpression of these heterologous proteins often causes an imbalance in cell equilibrium that results in misfolding and aggregation of recombinant proteins as inclusion bodies $[5,46]$. The increase of misfolded proteins and aggregates induces the E. coli HSR. Nevertheless, the induced HSR is usually not sufficient for high-speed production of proteins that are difficult to fold [5]. The use of synthetic biology strategies to regulate the HSR of $E$. coli can help to avoid protein aggregation, increase protein solubility, and increase the yield of correctly folded recombinant target proteins. For example, overexpression of the heterologous protein in combination with chaperones (DnaK/DnaJ/GrpE, GroEL/GroES, ClpB) or $\sigma^{32}$ proved to be effective in several studies because this enhanced protein folding, solubility, and activity $[46,47]$. IbpA and IbpB, two small HSPs, are also known to interact with 
the disaggregation and folding machinery of the cell [48]. The presence of both proteins slows protein misfolding and maintains partially folded proteins at the surface of the aggregate. This is crucial for efficient stabilization and disaggregation. Their interplay increases the ability of the DnaK system to refold proteins trapped in aggregates [48]. Therefore, manipulating the expression of HS genes using synthetic biology strategies can help to fine-tune the production of heterologous proteins in E. coli.

However, although overexpression of the heterologous proteins in combination with chaperones generally increases protein solubility, this may not apply to all chaperones. No universal chaperone system is effective in every case because they often handle specific substrates, and different chaperones should therefore be tested to determine efficient protein folding [49,50]. Owing to the important role of chaperones in heterologous production, TaKaRa Bio commercializes a chaperone plasmid set (Cat. 3340-TK) that includes five plasmids for chaperone expression in E. coli. The plasmids allow coexpression of the E. coli HSP chaperones GroES, GroEL, DnaK, DnaJ, and GrpE in different combinations, and these have been used in several studies to increase the recovery of expressed proteins in the soluble fraction [51-53]. Zhang and colleagues [50] used a different approach to increase recombinant protein production. They reprogrammed $E$. coli proteostasis network by co-overexpressing a $\sigma^{32}$ mutant [54] that is insensitive to negative feedback regulation by the DnaK and GroE systems (Box 1). The increased stability of the mutant allows a non-transient HSR to be maintained. In addition, in this method it is not necessary to study which chaperone system is most suitable to increase recombinant protein solubility. Cho and colleagues [55] compared the overexpressing $\sigma^{32}$ mutant to co-overexpressing DnaK or GroE systems, and verified that overexpression of the $\sigma^{32}$ mutant allows increased recombinant protein folding. In the future, the use of synthetic biology tools such as gene-editing technology to modify the $\sigma^{32}$ gene in the $E$. coli genome could be an interesting approach to increase recombinant protein production.

In addition to increasing recombinant proteins solubility and activity, chaperones can be cooverexpressed to engineer a stress response system capable of increasing $E$. coli tolerance to solvents. For example, the co-overexpression of GroES, GroEL, GrpE, and ClpB increased tolerance to toxic chemicals such as butanol and ethanol $[56,57]$. IbpA overexpression also improved isopentenol yields [58].

Chaperones have also been overexpressed in E. coli, purified, and immobilized on a chromatography column to refold apparently irreversibly denatured proteins, to renature insoluble proteins from an inclusion body, or to recondition enzymes that have lost their activity because of long storage [59]. GroEL is the most commonly used chaperone for in vitro refolding [59-61]. GroES in combination with GroEL, or with folding catalysts such as protein disulfide isomerase and peptidyl-prolyl isomerase, also proved to be beneficial [59]. Although the application of chaperones on an large scale is limited, the chaperone systems proved to be stable and maintain biological activity even after long storage [59,60], and some patents on this topic have been filed [62].

In addition to upregulating HSP genes, HS promoters may also be useful for producing heterologous compounds (Figure 2) in synthetic biology applications. Chemical inducers such as IPTG or anhydrotetracycline (aTc) are commonly used to trigger protein expression in smallscale fermentations $[63,64]$. However, their use should be avoided in large-scale fermentations because, as previously stated, they can be expensive and can lead to safety problems because chemical inducers are potentially toxic $[8,22,29]$. Therefore, their presence in waste effluents or as contaminants in the final purified recombinant product must be eliminated. This concern is 
even greater in the production of pharmaceutical-grade proteins and other products for human or animal use [65]. Furthermore, the number of inducible promoters is limited and, because the complexity of metabolic pathways is increasing, new inducible promoters will be necessary to control specific elements of each pathway [66-68]. Therefore, the usefulness of HS promoters to trigger the expression of novel biological parts, networks, and pathways has been evaluated $[13,17,65,69]$. Temperature induction is a very simple inducible system because increasing/ decreasing the temperature of the medium is technically easily achievable, safe, fast and cheap. In addition, the use of heat may simplify downstream processing because it minimizes culture handling and contamination risks [65]. The heat transfer limitations of bioreactors should be considered, but slow heating rates such those often observed in large-scale bioreactors may favor heterologous protein production [69]. Fast heating rates generate a large imbalance between tricarboxylic acid cycle and glycolysis, leading to high energy requirements for overcoming the physiological stress $[69,70]$. In addition, HS promoters can help to reduce inclusion body formation and proteolytic degradation of more complex proteins because they are in general weaker promoters, and therefore the synthesis rate of the recombinant protein is reduced, allowing proper folding. HS promoters may also be used in a synthetic circuit that couples the control of recombinant protein production to a stress-induced, negative feedback mechanism [41]. This circuit allows the cell to shut down recombinant protein production when stress signals are detected. For that purpose, synthetic biology principles, such as the integration of standardized parts in a synthetic circuit, have been used. For example, a target recombinant protein was expressed from an inducible bacteriophage T7 promoter engineered to be repressed by TetR expressed from the stress-sensitive ibpA promoter; this led to downregulation of protein expression following cell stress induced by accumulation of the recombinant protein [41].

Therapeutic Applications

The human HSR has been studied for potential applications in gene therapy owing to its efficiency and its potential to be induced by an external heat source [71]. Controlling gene expression in gene therapy is crucial for managing the timing and location of drug production because minimizing systemic toxicity is vital [72]. Hyperthermia (a procedure that increases the body or local temperature above $37^{\circ} \mathrm{C}$ ) [73] can be applied in a well-defined volume of tissue using appropriate heating techniques. Several studies have demonstrated the feasibility a delivering a therapeutic gene product controlled in space and time and activated by a minimally invasive heating method using a HS promoter $[71,74]$. The development of a novel gene therapy to treat cancer cells in which therapeutic genes are activated by heat seems a logical approach because hyperthermia is currently often used in combination with chemotherapy and radiotherapy [73]. For instance, by introducing into cancer cells a suicide gene under the control of a HSP promoter, selective reduction or even elimination of the tumor was observed [75]. Heat-inducible gene expression was also combined with stem cell delivery to permeabilize in a non-invasive way the blood-brain barrier with spatiotemporal precision [76]. This method may be used in the future to deliver different therapeutic drugs and to facilitate the treatment of central nervous system diseases.

Likewise, E. coli HS promoters may also have important roles in healthcare applications using synthetic biology approaches. Using synthetic biology, bacteria can be engineered for therapeutic applications such as gene delivery vectors, vaccines, or to sense the environment and generate a biological response, for example the release of a specific drug [13,68,77-81]. Bacteria could be engineered to produce a required compound in situ, and the expression of specific biological parts or pathways could be triggered by a temperature increase [82]. This increase could occur by combining the bacterial therapy with, for example, laser or focused 
ultrasound treatments [82] that are often used to treat solid tumors and that increase the local body temperature. This in situ production could be highly beneficial in the cases where the drug has low bioavailability (e.g., curcumin) $[12,13,83,84]$. Future microbial therapy applications may also include the ability to detect fever in the host and reduce the response to inflammation by releasing a therapeutic agent [82]. In addition, temperature-dependent killing switches may be used to prevent the survival of administered microbes after the desired treatment [82]. This type of containment strategy [85] can decrease the safety concerns of using $E$. coli as a vector in the clinic.

The $E$. coli HSR mechanism can also be used to detect novel $\beta$-lactam antibiotics in the environment [86]. For example, the E. coli grpE promoter was fused to the lux reporter and, when multi-resistant strains were exposed to antibiotics to which they were not resistant, the stress response was triggered and bioluminescence was detected. Moreover, chaperone overexpression could be very promising in treatments for diseases associated with protein aggregation such as Alzheimer's or Huntington's $[87,88]$. Therefore, research has aimed at understanding how chaperones with a role in protein disaggregation (disaggregases) function in $E$. coli with a view to applying that knowledge to the treatment of human disease $[89,90]$.

\section{Concluding Remarks and Future Perspectives}

The E. coli HSR involves a large number of complex controls that integrate multiple different signals to precisely regulate the amounts of all the proteins involved. These controls can minimize cellular requirements for HSPs and save energy. Therefore, they can be very useful in synthetic biology to create parts, devices, and systems that can be used in different applications. Although extensive research is still required before hyperthermia-regulated gene therapy using E. coli HS promoters can be combined with different treatments, other potential applications are not far from becoming a reality. For example, the creation of biosensors to detect chemical pollutants in water using synthetic biology has received a great amount of attention in recent years. The combination of a HS promoter and a reporter with a colorimetric output can be used to create a cheap and practical device for use in developing countries. The use of HS promoters in industry can also bring several advantages, as well as the coexpression of chaperones to increase recombinant protein folding, solubility, secretion, and final yield. Overall, the use of the E. coli HSR mechanism in synthetic biology approaches holds great promise even though many challenges remain (see Outstanding Questions).

\section{Acknowledgments}

This study was supported by the Portuguese Foundation for Science and Technology (FCT) under the scope of the strategic funding of UID/BIO/04469/2013 unit and COMPETE 2020 (POCl-01-0145-FEDER-006684) and under the scope of the Project RECI/BBB-EBI/0179/2012 (FCOMP-01-0124-FEDER-027462). The authors also acknowledge financial support from BioTecNorte operation (NORTE-01-0145-FEDER-000004) funded by the European Regional Development Fund under the scope of Norte2020-Programa Operacional Regional do Norte, and a Postdoctoral grant (UMINHO/BPD/37/2015) to J.L.R. funded by the FCT.

\section{References}

1. Zhao, K. et al. (2005) The global transcriptional response of Escherichia coli to induced $\sigma^{32}$ protein involves $\sigma^{32}$ regulon activation followed by inactivation and degradation of $\sigma^{32}$ in vivo. J. Biol. Chem. 280, 17758-17768

2. Berney, M. et al. (2006) Specific growth rate determines the sensitivity of Escherichia coli to thermal, UVA, and solar disinfection. Appl. Environ. Microbiol. 72, 2586-2593

3. Eltzov, E. et al. (2015) Creation of a new portable biosensor for water toxicity determination. Sens. Actuators B Chem. 221, 1044-1054
4. Müller, A. et al. (2013) Nonnative disulfide bond formation activates the $\sigma 32$-dependent heat shock response in Escherichia coli. J. Bacteriol. 195, 2807-2816

5. Li, Z. et al. (2017) Global proteome response of Escherichia coli BL21 to production of human basic fibroblast growth factor in complex and defined medium. Eng. Life Sci. 17, 881-891

6. Carroni, M. et al. (2014) Head-to-tail interactions of the coiledcoil domains regulate $\mathrm{ClpB}$ activity and cooperation with $\mathrm{Hsp} 70$ in protein disaggregation. Elife 3, e02481

\section{Outstanding Questions}

The E. coli strains used in synthetic biology are commensal or non-pathogenic. However, they may develop into pathogenic variants under some environmental conditions. Hence, could $E$. coli in the future be considered for biomedical applications such as living vaccines, in situ treatments, or in situ diagnosis - or can this host only be used to establish proof-of-principle? Will the public or regulatory agencies as the FDA accept the use of this microorganism, or will the use of probiotic strains be imperative?

The strengths of HS promoters can be difficult to adjust. Therefore, can these promoters be used when very precise control of complex metabolic pathways is needed? Will the level of induction be sufficient or suitable for the different applications? 
7. Guo, M.S. and Gross, C.A. (2014) Stress-induced remodeling of the bacterial proteome. Curr. Biol. 24, R424-R434

8. Hoynes-O'Connor, A. and Moon, T.S. (2015) Programmable genetic circuits for pathway engineering. Curr. Opin. Biotechnol. 36, 115-121

9. Seo, S.W. et al. (2013) Synthetic biology: tools to design microbes for the production of chemicals and fuels. Biotechnol. Adv. 31, 811-817

10. Jullesson, D. et al. (2015) Impact of synthetic biology and metabolic engineering on industrial production of fine chemicals. Biotechnol. Adv. 33, 1395-1402

11. Hoynes-O'Connor, A. and Moon, T.S. (2016) Development of design rules for reliable antisense RNA behavior in E. coli. ACS Synth. Biol. 5, 1441-1454

12. Rodrigues, L.R. and Kluskens, L.D. (2011) Synthetic biology \& bioinformatics prospects in the cancer arena. In Computational Biology and Applied Bioinformatics (Lopes, H.S. and Cruz, L.M., eds), pp. 159-186, InTech

13. Rodrigues, J.L. et al. (2017) Hydroxycinnamic acids and curcumin production in engineered Escherichia coli using heat shock promoters. Biochem. Eng. J. 125, 41-49

14. Nonaka, G. et al. (2006) Regulon and promoter analysis of the E. coli heat-shock factor, $\sigma^{32}$, reveals a multifaceted cellular response to heat stress. Genes Dev. 20, 1776-1789

15. Wade, J.T. et al. (2006) Extensive functional overlap between $\sigma$ factors in Escherichia coli. Nat. Struct. Mol. Biol. 13, 806-814

16. Rasouly, A. et al. (2009) YbeY, a heat shock protein involved in translation in Escherichia coli. J. Bacteriol. 191, 2649-2655

17. Rodrigues, J.L. et al. (2014) Selection of Escherichia coli heat shock promoters towards their application as stress probes. $J$. Biotechnol. 188, 61-71

18. Richmond, C.S. et al. (1999) Genome-wide expression profiling in Escherichia coli K-12. Nucleic Acids Res. 27, 3821-3835

19. Gaubig, L.C. et al. (2011) Multiple layers of control govern expression of the Escherichia coli ibpAB heat-shock operon. Microbiology 157, 66-76

20. Dahl, R.H. et al. (2013) Engineering dynamic pathway regulation using stress-response promoters. Nat. Biotechnol. 31, 10391046

21. Hoynes-O'Connor, A. et al. (2015) De novo design of heatrepressible RNA thermosensors in E. coli. Nucleic Acids Res. 43, 6166-6179

22. Kortmann, J. and Narberhaus, F. (2012) Bacterial RNA thermometers: molecular zippers and switches. Nat. Rev. Microbiol. 10, 255-265

23. Krajewski, S.S. and Narberhaus, F. (2014) Temperature-driven differential gene expression by RNA thermosensors. Biochim. Biophys. Acta 1839, 978-988

24. Saragliadis, A. et al. (2013) Thermozymes: synthetic RNA thermometers based on ribozyme activity. RNA Biol. 10, 1009-1016

25. Klinkert, B. et al. (2012) Thermogenetic tools to monitor temperature-dependent gene expression in bacteria. J. Biotechnol. $160,55-63$

26. Roßmanith, J. and Narberhaus, F. (2016) Exploring the modular nature of riboswitches and RNA thermometers. Nucleic Acids Res. 44, 5410-5423

27. Lioliou, E. et al. (2010) RNA-mediated regulation in bacteria: from natural to artificial systems. New Biotechnol. 27, 222-235

28. Neupert, J. and Bock, R. (2009) Designing and using synthetic RNA thermometers for temperature-controlled gene expression in bacteria. Nat. Protoc. 4, 1262-1273

29. Gupta, S.K. and Shukla, P. (2016) Advanced technologies for improved expression of recombinant proteins in bacteria: perspectives and applications. Crit. Rev. Biotechnol. 36, 10891098

30. Waldminghaus, T. et al. (2008) Generation of synthetic RNAbased thermosensors. Biol. Chem. 389, 1319-1326

31. Sen, S. et al. (2017) Design of a toolbox of RNA thermometers. ACS Synth. Biol. 6, 1461-1470
32. Padilla-Martínez, F. et al. (2015) Cell damage detection using Escherichia coli reporter plasmids: fluorescent and colorimetric assays. Arch. Microbiol. 197, 815-821

33. Cha, H.J. et al. (1999) Green fluorescent protein as a noninvasive stress probe in resting Escherichia coli cells. Appl. Environ. Microbiol. 65, 409-414

34. Kotova, V.Y. et al. (2010) Lux-biosensors for detection of SOSresponse, heat shock, and oxidative stress. Appl. Biochem. Microbiol. 46, 781-788

35. Chugunova, E. et al. (2015) Synthesis and antimicrobial activity of novel structural hybrids of benzofuroxan and benzothiazole derivatives. Eur. J. Med. Chem. 93, 349-359

36. Van Der Meer, J.R. and Belkin, S. (2010) Where microbiology meets microengineering: design and applications of reporter bacteria. Nat. Rev. Microbiol. 8, 511-522

37. Kraft, M. et al. (2007) An online monitoring system based on a synthetic sigma32-dependent tandem promoter for visualization of insoluble proteins in the cytoplasm of Escherichia coli. Appl. Microbiol. Biotechnol. 75, 397-406

38. Sagi, E. et al. (2003) Fluorescence and bioluminescence reporter functions in genetically modified bacterial senso strains. Sens. Actuators B Chem. 90, 2-8

39. Aertsen, A. et al. (2004) Heat shock protein-mediated resistance to high hydrostatic pressure in Escherichia coli. Appl. Environ. Microbiol. 70, 2660-2666

40. Nemecek, S. et al. (2008) Design of transcriptional fusions of stress sensitive promoters and GFP to monitor the overburden of Escherichia coli hosts during recombinant protein production. Bioprocess Biosyst. Eng. 31, 47-53

41. Dragosits, M. et al. (2012) A synthetic biology approach to selfregulatory recombinant protein production in Escherichia coli. J. Biol. Eng. 6, 1-10

42. Popovtzer, R. et al. (2005) Novel integrated electrochemica nano-biochip for toxicity detection in water. Nano Lett. 5 , 1023-1027

43. Sekhon, S.S. et al. (2014) Stress specific Escherichia coli biosensors based on gene promoters for toxicity monitoring. Mol. Cell. Toxicol. 10, 369-377

44. Eltzov, E. et al. (2011) Creation of a fiber optic based biosensor for air toxicity monitoring. Sens. Actuators B Chem. 155, 859867

45. Eltzov, E et al. (2015) Bioluminescent liquid light guide pad biosensor for indoor air toxicity monitoring. Anal. Chem. 87 , 3655-3661

46. Lee, A.R. et al. (2015) Soluble expression of the fucosyltransferase gene from Helicobacter pylori in Escherichia coli by co-expression of molecular chaperones. Microbiol. Biotechnol. Lett. 43, 213-219

47. Veisi, K. et al. (2015) Chaperone-assisted soluble expression of a humanized anti-EGFR ScFv antibody in E. coli. Adv. Pharm. Bull. 5 (Suppl. 1), 621

48. Ratajczak, E. et al. (2009) Distinct activities of Escherichia coli small heat shock proteins IbpA and IbpB promote efficient protein disaggregation. J. Mol. Biol. 386, 178-189

49. Jhamb, K. and Sahoo, D.K. (2012) Production of soluble recombinant proteins in Escherichia coli: effects of process conditions and chaperone co-expression on cell growth and production of xylanase. Bioresour. Technol. 123, $135-143$

50. Zhang, X. et al. (2014) Heat-shock response transcriptiona program enables high-yield and high-quality recombinant protein production in Escherichia coli. ACS Chem. Biol. 9, 19451949

51. Cai, R. et al. (2017) Improved soluble bacterial expression and properties of the recombinant flavonoid glucosyltransferase UGT73G1 from Allium cepa. J. Biotechnol. 255, 9-15

52. Welner, D.H. et al. (2017) Plant cell wall glycosyltransferases: high-throughput recombinant expression screening and genera requirements for these challenging enzymes. PLoS One 12 e0177591 
53. Kato, T. et al. (2017) Functional analysis of ribonucleotide reductase from Cordyceps militaris expressed in Escherichia coli. Appl. Biochem. Biotechnol. 182, 1307-1317

54. Yura, T. et al. (2007) Analysis of $\sigma^{32}$ mutants defective in chaperone-mediated feedback control reveals unexpected complexity of the heat shock response. Proc. Natl. Acad. Sci. U. S. A. 104, 17638-17643

55. Cho, Y. et al. (2015) Individual and collective contributions of chaperoning and degradation to protein homeostasis in E. coli. Cell Rep. 11, 321-333

56. Zingaro, K.A. and Papoutsakis, E.T. (2013) GroESL overexpression imparts Escherichia coli tolerance to i-, n-, and 2-butanol, 1,2,4-butanetriol and ethanol with complex and unpredictable patterns. Metab. Eng. 15, 196-205

57. Zingaro, K.A. and Papoutsakis, E.T. (2012) Toward a semisynthetic stress response system to engineer microbial solvent tolerance. MBio 3, e00308-12

58. Foo, J. et al. (2014) Improving microbial biogasoline production in Escherichia coli. MBio 5, e01932-14

59. Jhamb, K. et al. (2008) Immobilized chaperones: a productive alternative to refolding of bacterial inclusion body proteins. Process Biochem. 43, 587-597

60. Antonio-Pérez, A. et al. (2012) Chromatographic refolding of rhodanese and lysozyme assisted by the GroEL apical domain, DsbA and DsbC immobilized on cellulose. J. Chromatogr. A 1248, 122-129

61. Marchenko, N.Y. et al. (2016) Affinity chromatography of chaperones based on denatured proteins: analysis of cell lysates of different origin. Protein Expr. Purif. 119, 117-123

62. Eiberle, M.K. and Jungbauer, A. (2010) Technical refolding of proteins: do we have freedom to operate? Biotechnol. J. 5, 547559

63. Rodrigues, J. et al. (2015) Production of curcuminoids from tyrosine by a metabolically engineered Escherichia coli using caffeic acid as an intermediate. Biotechnol J. 10, 599-609

64. Rodrigues, J. et al. (2015) Heterologous production of caffeic acid from tyrosine in Escherichia coli. Enzyme Microb. Technol. $71,36-44$

65. Valdez-Cruz, N.A. et al. (2010) Production of recombinant proteins in E. coli by the heat inducible expression system based on the phage lambda $\mathrm{pL}$ and/or pR promoters. Microb. Cell Fact. 9, $1-16$

66. Rodrigues, J.L. and Rodrigues, L.R. (2017) Synthetic biology: perspectives in industrial biotechnology. In Foundations of Biotechnology and Bioengineering (Vol. 1): Current Developments in Biotechnology and Bioengineering (Pandey, A. and Teixiera, J., eds), pp. 239-269, Elsevier

67. Shive, E. and Prather, K.L. (2012) Synthetic biology devices as tools for metabolic engineering. Biochem. Eng. J. 65, 82-89

68. Rodrigues, J.L. et al. (2017) Synthetic biology strategies towards the development of new bioinspired technologies for medical applications. In Bioinspired Materials for Medical Applications (Rodrigues, L. and Mota, M., eds), pp. 451-497, Woodhead Publishing

69. Caspeta, L. et al. (2009) The effect of heating rate on Escherichia coli metabolism, physiological stress, transcriptional response, and production of temperature-induced recombinant protein: a scale-down study. Biotechnol. Bioeng. 102, 468-482

70. Caspeta, L. et al. (2013) Enhancing thermo-induced recombinant protein production in Escherichia coli by temperature oscillations and post-induction nutrient feeding strategies. I. Biotechnol. 167, 47-55

71. Eker, O.F. et al. (2011) Combination of cell delivery and thermoinducible transcription for in vivo spatiotemporal control of gene expression: a feasibility study. Radiology 258, 496-504

72. Forbes, N.S. (2010) Engineering the perfect (bacterial) cancer therapy. Nat. Rev. Cancer 10, 785-794

73. Walther, W. and Stein, U. (2009) Heat-responsive gene expression for gene therapy. Adv. Drug Deliv. Rev. 61, 641-649
74. Fortin, P.-Y. et al. (2015) Spatiotemporal control of gene expres sion in bone-marrow derived cells of the tumor microenvironment induced by MRI guided focused ultrasound. Oncotarget 6 , 23417-23426

75. Luo, J. et al. (2016) Radiofrequency hyperthermia promotes the therapeutic effects on chemotherapeutic-resistant breast cancer when combined with heat shock protein promoter-controlled HSV-TK gene therapy: toward imaging-guided interventional gene therapy. Oncotarget 7, 65042

76. Xiong, X. et al. (2015) Remote spatiotemporally controlled and biologically selective permeabilization of blood-brain barrier. J. Control. Release 217, 113-120

77. Anderson, J.C. et al. (2006) Environmentally controlled invasion of cancer cells by engineered bacteria. J. Mol. Biol. 355, 619627

78. Dai, Y. et al. (2013) Construction of an inducible cell-communication system that amplifies Salmonella gene expression in tumor tissue. Biotechnol. Bioeng. 110, 1769-1781

79. Garmory, H.S. et al. (2003) The use of live attenuated bacteria as a delivery system for heterologous antigens. J. Drug Target. 11 , 471-479

80. Shankar, S. and Pillai, M.R. (2011) Translating cancer research by synthetic biology. Mol. Biosyst. 7, 1802-1810

81. Swofford, C.A. et al. (2015) Quorum-sensing Salmonella selectively trigger protein expression within tumors. Proc. Natl. Acad. U. S. A. $112,3457-3462$

82. Piraner, D.I. et al. (2017) Tunable thermal bioswitches for in vivo control of microbial therapeutics. Nat. Chem. Biol. 13, 75-80

83. Rodrigues, J.L. et al. (2015) Heterologous production of curcuminoids. Microbiol. Mol. Biol. Rev. 79, 39-60

84. Couto, M.R. et al. (2017) Optimization of fermentation conditions for the production of curcumin by engineered Escherichia coli. J. R. Soc. Interface 14, 1-8

85. Chan, C.T. et al. (2016) 'Deadman' and 'passcode' microbial kill switches for bacterial containment. Nat. Chem. Biol. 12, 82

86. Eltzov, E. et al. (2012) Multi-resistance as a tool for detecting novel beta-lactam antibiotics in the environment. Sens. Actuators B Chem. 174, 342-348

87. Iram, A. and Naeem, A. (2014) Protein folding, misfolding, aggregation and their implications in human diseases: discovering therapeutic ways to amyloid-associated diseases. Cell Biochem. Biophys. 70, 51-61

88. Doyle, S.M. et al. (2013) Protein rescue from aggregates by powerful molecular chaperone machines. Nat. Rev. Mol. Cell Biol. 14, 617-629

89. Johnston, D.M. et al. (2017) Substrate discrimination by ClpB and Hsp104. Front. Mol. Biosci. 4, 1-12

90. Gasset-Rosa, F. et al. (2014) Direct assessment in bacteria of prionoid propagation and phenotype selection by Hsp70 chaperone. Mol. Microbiol. 91, 1070-1087

91. Guisbert, E. et al. (2008) Convergence of molecular, modeling and systems approaches for an understanding of the Escherichia coli heat shock response. Microbiol. Mol. Biol. Rev. 72 545-554

92. Arsène, F. et al. (2000) The heat shock response of Escherichia coli: Review. Int. J. Food Microbiol. 55, 3-9

93. Miyazaki, R. et al. (2016) A novel SRP recognition sequence in the homeostatic control region of heat shock transcription factor б32. Sci. Rep. 6, 24147

94. Lim, B. et al. (2013) Heat shock transcription factor $\sigma 32$ co-opts the signal recognition particle to regulate protein homeostasis in E. coli. PLoS Biol. 11, e1001735

95. Xu, X. et al. (2015) Heat shock transcription factor $\delta 32$ is targeted for degradation via an ubiquitin-like protein ThiS in Escherichia coli. Biochem. Biophys. Res. Commun. 459, 240-245

96. Oguchi, Y. et al. (2012) A tightly regulated molecular toggle controls AAA+ disaggregase. Nat. Struct. Mol. Biol. 19, 1338-1346 
97. Treviño-Quintanilla, L.G. et al. (2013) Anti-sigma factors in E. coli: common regulatory mechanisms controlling sigma factors availability. Curr. Genom. 14, 378-387

98. Okuno, T. and Ogura, T. (2013) FtsH protease-mediated regulation of various cellular functions. In Regulated Proteolysis in Microorganisms (Dougan, D.A., ed.),

pp. 53-69, Springer
99. Meyer, A.S. and Baker, T.A. (2011) Proteolysis in the Escherichia coli heat shock response: a player at many levels. Curr. Opin. Microbiol. 14, 194-199

100. Obrist, M. et al. (2009) Region C of the Escherichia coli heat shock sigma factor $\mathrm{RpoH}$ (sigma 32) contains a turnover element for proteolysis by the FtsH protease. FEMS Microbiol. Lett. 290, 199-208 